\title{
Resonant absorption in randomly driven coronal loops
}

\author{
Anik De Groof and Marcel Goossens \\ Centre for Plasma-Astrophysics, K.U. Leuven, Celestijnenlaan 200B, 3001 Leuven, Belgium
}

\begin{abstract}
The efficiency of resonant absorption as heating mechanism is investigated in footpoint driven loops. From earlier results [3] we know that the input energy of radially polarized motions is mainly stored in fast body modes, which can resonantly couple to Alfvén waves. The coronal loop is driven by quasi-random footpoint motions in order to simulate realistic pulses. While in harmonically driven models the loop was only heated at one single layer, in the present study multiple resonant layers are found, resulting in a more globally heated loop.
\end{abstract}

\section{Introduction}

Magnetosonic waves and Alfvén waves in coronal loops can be triggered either by fast motions of the photospheric footpoints or more likely by reconnection events in smaller loops. Observational evidence of MHD waves propagating in coronal loops has indeed been reported [5]. Tataronis \& Grossmann[8] were the first to give a basic theory on plasma heating by the Alfvén continuum waves. In 1978, Ionson[4] suggested that resonant absorption might effectively heat the solar corona. A lot of work was done on sideways excitation of these resonant Alfvén waves ([2] for references). Later studies focussed on different kinds of footpoint motions in order to excite resonant Alfvén waves either directly by azimuthally polarised footpoint motions or indirectly by radially polarised footpoint motions. Berghmans et al.[1] and Tirry et al.[9] revealed the importance of the presence of quasi-modes in both cases.

So far, most of the modelled footpoint motions were harmonic or consisted of one pulse. In the present paper we want to figure out how the results alter for quasi-randomly driven loops. In [3], we studied the behaviour of the fast waves within the loop without coupling to Alfvén waves and found that driving at the loop's feet forms a good basis for resonant absorption as heating mechanism. In the present paper, we report on the efficiency of resonant absorption in the coupled case $\left(k_{y} \neq 0\right)$, where the excited body waves can couple to Alfvén waves, and present some future plans.

\section{Model}

The coronal loop is modelled as a static, straight, gravitationless plasma slab with thickness $b$, obeying the standard set of linear, ideal MHD equations. In our Cartesian coordinate system, the $x$-coordinate corresponds to the direction of the inhomogeneity in the equilibrium, the $y$-coordinate is the (ignorable) azimuthal coordinate and the $z$-coordinate 
represents the direction along the loop. At $z=0$ we impose radially polarised, smallamplitude perturbations whereas at $z=L$ we assume the loop to be line-tied (see [3] for more details). The plasma is permeated by a uniform magnetic field $\left(\mathbf{B}_{\mathbf{0}}=B_{0} \mathbf{e}_{\mathbf{z}}\right)$ and has a uniform pressure $p_{0}$ which we neglect in comparison with the magnetic pressure. The inhomogeneity of the plasma is introduced by a continuously varying density $\rho_{0}(x)=\rho_{A}+\rho_{B} \cos \left(\frac{\pi}{b} x\right)$ with $\rho_{B}<\rho_{A}$, which models the higher density inside the loop. Since the equilibrium quantities are constant in the $y$-coordinate which runs over an infinite interval, we can Fourier analyse with respect to $y$. For the Fourier component corresponding to wave number $k_{y}$, the time evolution and the spatial variation in $x$ and $z$ are described by

$$
\left\{\frac{1}{v_{A}^{2}} \frac{\partial^{2}}{\partial t^{2}}-\frac{\partial^{2}}{\partial z^{2}}-\frac{\partial^{2}}{\partial x^{2}}\right\} \xi_{x}=i k_{y} \frac{\partial \xi_{y}}{\partial x} \quad \text { and } \quad\left\{\frac{1}{v_{A}^{2}} \frac{\partial^{2}}{\partial t^{2}}-\frac{\partial^{2}}{\partial z^{2}}+k_{y}^{2}\right\} \xi_{y}=i k_{y} \frac{\partial \xi_{x}}{\partial x}
$$

with the Lagrangian displacement $\boldsymbol{\xi}$ and the Alfvén speed $v_{A}(x)=B_{0} / \sqrt{\rho_{0}(x)}$.

This coupled system of partial differential equations in $\xi_{x}$ and $\xi_{y}$ describes the coupled fastAlfvén waves. Slow waves are absent $\left(\xi_{z}=0\right)$ because the plasma pressure is neglected. Since we focus on radially polarised footpoint motions, only fast waves are driven directly. When $k_{y} \neq 0$, some of these fast MHD waves, the quasi-modes, couple to Alfvén waves at the resonant surfaces where the ideal Alfvén wave resonance condition is satisfied. Length, speed, magnetic field strength and density are non-dimensionalised with respect to $b, v_{A}(0), B_{0}$ and $\rho(0)$ respectively.

The mathematical approach in this paper is based on the method used in [9]. The footpoint motions are represented by inhomogeneous boundary conditions for (1) at the $z=0$ and $z=L$ boundary planes: $\xi_{x}=R(x) T(t)$ at $z=0$ and zero at $z=L ; \xi_{y}$ is zero at both boundaries. Further we assume that the dependencies on $x$ and $t$ of the footpoint motions are separable and that $\xi_{x}, \xi_{y}$ and both their time derivatives are zero at $t=0$. A convenient way to solve the coupled partial differential equations (1) is to get rid of as many derivatives as possible, as described in detail by [9]. This approach enables us to obtain a solution for fast and Alfvén waves written as a superposition of eigenmodes (see [3]). Since, for small values of $k_{y}$, the global compressional oscillations are dominated by poloidal perturbations, and the Alfvénic disturbances are dominated by a toroidal polarisation, we use the term fast mode energy for the combined poloidal and compressional energy and the term Alfvén mode energy for the toroidal energy.

\section{Efficiency of resonant absorption}

For $k_{y}=0$ and $R=0$, expressions (1) form two separate eigenvalue problems for fast and Alfvén eigenmodes; for $k_{y} \neq 0$ the equations are coupled. In the latter case, incident fast waves can resonantly excite a local Alfvén continuum wave if their frequency matches the local Alfvén frequency. From De Groof et al. [3], we know that most of the input energy is converted to body mode energy (around 95\%) so that the energy leakage is minimal. For resonant absorption to be efficient as heating mechanism, several criteria have to be satisfied. First, we need an efficient energy transfer from the footpoint motions to the Alfvén waves (via the fast waves). Second, the resonances built up that way should produce the necessary small length scales needed for dissipation in the corona. As a third criteria, the time scales should be small compared to the lifetime of the loop and finally we check whether resonant absorption can heat the loop globally. 


\subsection{Time evolution of fast and Alfvén waves}

For simplicity, we take the loop dimensions to be $L=1$ and $b=1$; the density parameters $\rho_{A}$ and $\rho_{B}$ are 0.6 and 0.4 respectively. The footpoint motions are modelled as a succession of pulses with the following time and $x$ dependencies:

$$
\begin{aligned}
& T(t)= \begin{cases}\sin \left(a t-\frac{\pi}{2}\right)+1 & \text { as } 0 \leq t \leq \frac{2 \pi}{a}, \\
0 & \text { as } t>\frac{2 \pi}{a}\end{cases} \\
& R(x)=\sin (\pi x)
\end{aligned}
$$

which are chosen in order to simulate instant 'kicks' at the loop's feet. From Fig. 1, we conclude that after the driving has stopped, the fast mode energy is transferred to Alfvén mode energy. The efficiency of this transfer depends on several parameters, like the azimuthal wavenumber $k_{y}$, which acts as a coupling parameter between fast modes

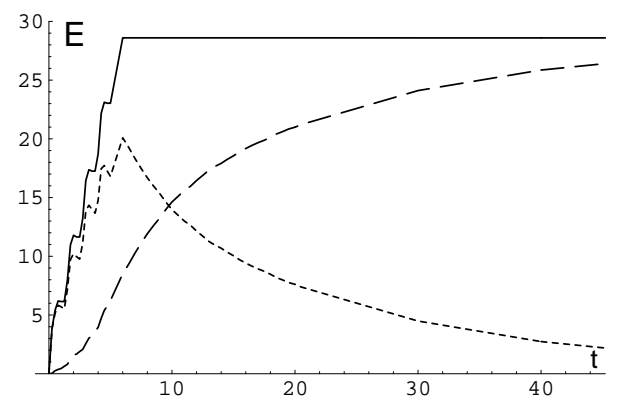

Figure 1: Time evolution of $E_{\text {fast }}$ (dotted), $E_{\text {Alfvén (dashed) and } E_{\text {tot }}}$ (solid) after 5 identical pulses. and Alfvén modes, and the duration of the pulses.

\subsection{Creation of small length scales}

For the resonant absorption to be valid and to be efficient, resonances have to be formed in the coronal loop within half of the loop's lifetime. Tirry et al.[9] demonstrated that when the loop is driven harmonically, resonance peaks are formed at the magnetic surfaces where the local Alfvén frequency equals the quasi-mode frequencies which are present in the driving.

As a first step to a real random driving, we drive the loop by a series of 30 pulses with randomly distributed widths and randomly distributed time intervals in between the pulses. The individual pulses have a time-dependence $\sin \left(a t-\frac{\pi}{2}\right)+1$ with pulse widths varying between 0.4 and $2.1(3<a<16)$ and time intervals smaller than 1 . As shown in Fig.2, indeed resonances are built up at the magnetic surfaces corresponding to the quasi-modes which

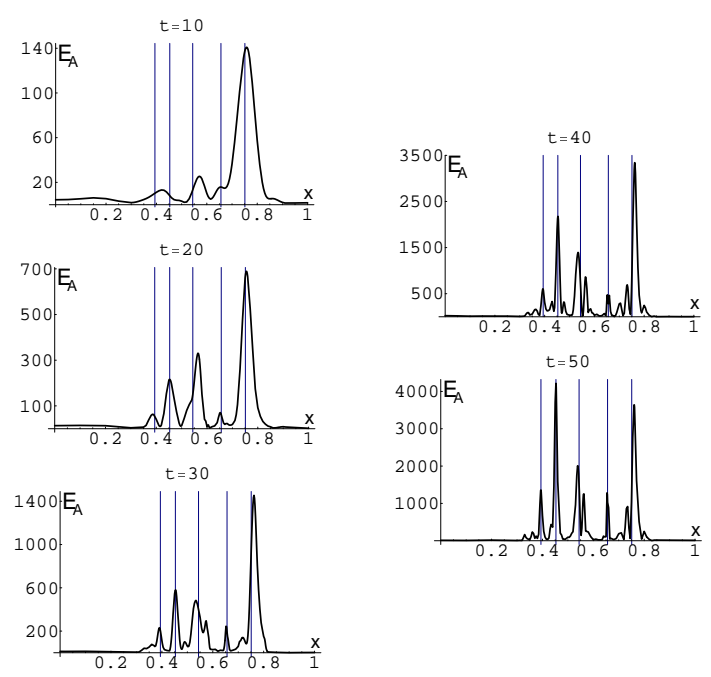

Figure 2: Alfvén energy as function of $x$ for $t=10$ to $t=50$ after driving with $a$ randomly varying pulse train. are excited by the driver (vertical lines). The modes corresponding to the first eigenfunctions and small values of $k_{z}$ are the most dominant (see [2]). In contrast to previous studies, where the heating was highly concentrated in a thin layer of the loop, our results show multiple resonant peaks and consequently a more globally heated loop. This difference is due to 2 main reasons. First, most of the earlier studies assumed monochromatic footpoint motions. In De Groof et al. 2000 [2], the importance of a more realistic driver is pointed out by comparing our results with those for an harmonic driver in the same model. In Fig. 3 the Alfvén mode energy excited 
by a monochromatic driver is plotted; qualitatively the graph is comparable to Fig. 2 $(t=40)$. Only one resonant peak is built up resulting from a fast-Alfvén mode energy transfer, namely at $x_{A}=0.54$. The second resonant peak, built up at the magnetic surface $x=0.59$ where the local Alfvén frequency equals the driving frequency, is forced by the harmonic driving and will not appear in more realistic, random motions. Another very important difference with previous studies which included a broadband driver but nevertheless had only a single heated magnetic shell, is the fact that all the quasi-modes are taken into account. Assuming $k_{z}$ to be constant, as done in many papers, excludes all but the first harmonic of the fast modes in the driving spectrum. As seen in Fig. 2, higher harmonics also lead to resonant peaks in the loop and consequently should not be excluded in studies of coronal heating.

Finally, resonant absorption can only be a viable coronal heating mechanism if the generation of the small length scales takes place on time scales shorter than the life time of coronal loops, which varies from

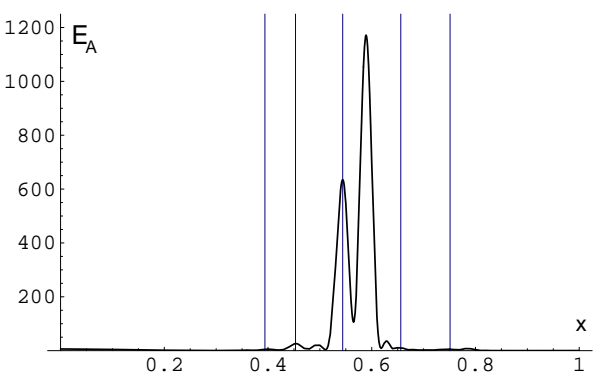

Figure 3: $E_{\text {Alfvén }}$ as a function of $x$ after an harmonic driving of 30 sine-pulses with period $2 \pi / 9$.

6 tot 24 hours. As calculated in [2], even for the slowest decrease, the time needed to generate length scales of $100 \mathrm{~m}$ lies around 3 hours. In addition Ofman \& Davila [6] showed that nonlinearity would lead to even smaller time scales by means of turbulence.

\section{Conclusions}

Resonant absorption in a linear, ideal MHD loop model, driven by quasi-random footpoint motions seems to be a viable dissipation mechanism. When only fast waves are driven, Alfvén waves are excited in an efficient way and resonances are built up at several surfaces in the loop. The implementation of a random driver and varying $k_{z}$ turns out to be very important since only then multiple resonant peaks are built on realistic time scales. In future work we would like to extend this analysis by taking into account the chromospheric coupling. Density profiles will then become time dependent [7] and we expect the resonant surfaces to shift in time which will result in an (optically) globally heated loop!

\section{References}

[1] Berghmans, D., and Tirry, W.J., $A$ E $A$ 325, 318-328 (1997)

[2] De Groof, A., and Goossens, M., $A \mathscr{G} A$ 356, 724-734 (2000)

[3] De Groof, A., Tirry, W.J., and Goossens, M., $A \mathscr{E} A$ 335, 329-340 (1998)

[4] Ionson, J.A. ApJ 226, 650-673 (1978)

[5] Nakariakov, V.M., Ofman, L., DeLuca, E.E., et al., Science 285, 862-864 (1999)

[6] Ofman, L., and Davila, J.M., J. Geophys. Res. 100, 23427 (1995)

[7] Ofman, L., Klimchuk, J.A., and Davila, J.M., ApJ 493, 474-479 (1998)

[8] Tataronis, J.A., and Grossmann, W., J. Plasma Phys. 13, 87 (1975)

[9] Tirry, W.J., Berghmans, D., and Goossens, M., A $\mathscr{G} A$ 322, 329 (1997) 